\title{
Etude Comparative entre les Composites à Base Plastique et l'Acier dans des Pièces Structurales de
} l'automobile

\section{Comparative Study between Plastic Composites and Steel in Structural}

\author{
Parts of Automobile
}

\author{
Abdelilah Elbazze ${ }^{1}$, Bouchaïb Radi ${ }^{2}$ \\ ${ }^{1}$ Laboratoire d'Ingénierie, Management Industriel et Innovation (IM2I) FSTS, Maroc elbazze.abdelilah@gmail.com \\ ${ }^{2}$ Laboratoire d'Ingénierie, Management Industriel et Innovation (IM2I) FSTS, Maroc, bouchaib.radi@yahoo.fr
}

RÉSUMÉ. L'objectif de ce travail est de montrer que les matériaux composites à base plastiques renforcés par des fibres minéraux (verre ou carbone, etc.) utilisés dans des pièces structurales de l'automobile assemblées avec leur environnement via des liaisons boulonnées, sont capables de remplacer certaines pièces en acier. Tout en gardant que ces matériaux ayant des avantages industrielles et économiques; tel qu'ils permettent d'une part d'alléger la structure automobile pour moins de consommation des carburants et d'autre part de baisser le cout de fabrication pour plus de compétitivité dans le marché automobile. Les tâches qui ont été faites dans cette étude est de soumettre ces pièces composites aux mêmes sollicitations que l'acier, tout en jouant des nouveaux concepts pour qu'elles s'adaptent à leur environnement et répondent aux critères de fiabilité et de validation d'une voiture en homologation. Les résultats de simulations numériques montrent la capacité de ces matériaux pour remplacer l'acier dans certaines pièces structurales de l'automobile et ils représentent un choix stratégique dans l'industrie automobile au futur.

ABSTRACT. The objective of this study is to show that composite materials based on plastic reinforced with mineral fibers (glass or carbon, etc.) used in structural parts of the automobile, assembled with their environment via bolted connections, are able to replace some steel parts. While keeping these materials have industrial and economic advantages; as they permit on the one hand to lighten the automotive structure for less consumption of fuels and on the other hand to lower the cost of manufacture for more competitiveness in the automotive market. The tasks that have been done in this study is to submit these composite parts to the same stresses as steel, by using new concepts so that they adapt to their environment and reply to the criteria of reliability and validation's car approval. The results of numerical simulations show the ability of these materials to replace steel in structural parts of the automobile and they represent a strategic choice in the automotive industry of the future.

MOTS-CLÉS. Matériaux composites, assemblage boulonnée, automobile, simulation numérique, calcul dynamique, crash, dynamique rapide, polypropylène, fibres de verre, carbone.

KEYWORDS. Composite materials, bolted assembly, automotive, numerical simulation, dynamic calculation, crash, fast dynamics, polypropylene, fiberglass, carbon.

\section{Introduction}

Les matériaux composites sont de plus en plus utilisés dans l'industrie automobile, ferroviaire, aéronautique. Dans le domaine automobile, la course aux économies d'énergie et aux limitations d'émissions de gaz polluants crée un intérêt croissant de la part des constructeurs et des équipementiers pour l'utilisation des matériaux de faibles densités et une bonne tenue mécanique. En parallèle, les soucis de protéger les automobilistes mais aussi les piétons lors d'un choc, sont à l'origine de normes de sécurité de plus en plus sévères. Constitués des matériaux aux caractéristiques complémentaires, les composites apparaissent comme de bons candidats pour répondre à toutes ces exigences. Ils possèdent en effet une faible densité, des propriétés mécaniques élevées et de plus, présentent des propriétés intéressantes en terme d'absorption d'énergie. En général, dans l'industrie automobile, ces matériaux sont destinés à être utilisés dans des pièces de structure telles que les absorbeurs de chocs avant, ou encore sur des pièces destinées à travailler en (c) 2019 ISTE OpenScience - Published by ISTE Ltd. London, UK - openscience.fr 
fatigue telles que les lames de suspension. Par contre l'utilisation de ces matériaux dans des pièces de la carrosserie est très limitée dans l'industrie automobile. L'objectif de ce travail est de montrer que les matériaux composites thermoplastique représentent un choix stratégique pour remplacer l'acier dans certaines pièces structurale de l'automobile, en se basant dans notre étude sur la simulation numérique pour renforcer le choix des composites.

\section{Matériaux composites thermoplastiques}

Les matériaux composites thermoplastiques sont constitués principalement d'un renfort fibreux noyé dans une matrice polymère. Les fibres ont pour rôle d'améliorer significativement les propriétés thermomécaniques et dynamiques de la matrice plastique. Les matériaux composites combinent la résistance et la rigidité ainsi que la légèreté, la flexibilité et la résistance à la corrosion des plastiques. Ils sont particulièrement adaptés aux contraintes de l'automobile. Aujourd'hui les fibres de verre sont les plus utilisés dans l'industrie automobile grâce à ces propriétés physiques et son adaptation avec les matrices plastiques.

\subsection{Fibres de verre}

Le verre appartient au groupe des matériaux inorganiques non métalliques, il n'a donc pas une structure macromoléculaire linéaire comme la plupart des autres fibres mais plutôt une structure cristalline qui lui donne une grande rigidité. Les fibres de verre sont les éléments du matériau composite qui apportent généralement des propriétés mécaniques intéressantes. Les qualités acquises de fibres de verre sont : bonnes caractéristiques mécaniques, légèreté, résistance thermique, compactibilité avec les résines, adaptabilité aux procédés de mise en œuvre, faible prix.

\begin{tabular}{|c|c|c|c|c|c|c|}
\hline \multicolumn{7}{|c|}{ Caractéristiques mécanique des filaments vierges } \\
\hline & \multicolumn{6}{|c|}{ Type de verre } \\
\hline & $\mathbf{E}$ & D & $\mathbf{A}$ & C & $\mathbf{R}$ & $\mathrm{S}$ \\
\hline $\begin{array}{l}\text { Contrainte à la rupture en } \\
\text { traction }\left(\mathrm{MPa}_{\mathbf{P}}\right)\end{array}$ & 3200 à 3400 & 2500 & 3100 & 3300 & 4400 & 4600 \\
\hline $\begin{array}{l}\text { Module d'élasticité en } \\
\text { traction (GPa) }\end{array}$ & 72 à 73 & 55 & 71 & 70,3 & 86 & 87 \\
\hline $\begin{array}{l}\text { Taux d'allongement à la } \\
\text { rupture en traction (\%) }\end{array}$ & 4,6 à 4,8 & 4,5 & 4,4 & 4,8 & 5,2 & 5,4 \\
\hline
\end{tabular}

Tableau 1. Caractéristiques mécanique mesurées sur des filaments vierges

\subsection{Matrices thermoplastiques}

Les composites que nous allons comparer avec l'acier, les propriétés mécaniques de la matrice sont généralement très faibles devant celles des renforts. Les courbes ci-dessous montrent clairement cette différence entre la matrice et les fibres : 


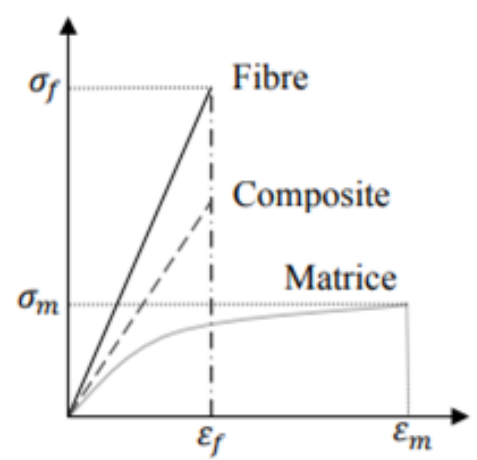

Figure 1. Courbes schématiques contrainte / déformation des différents constituants d'un composite unidirectionnel sollicité en traction longitudinale : $\varepsilon_{f}<\varepsilon_{m}$

Les résines thermoplastiques apparaissent généralement sous la forme de poudres ou de granulés qui doivent être chauffés pour être mis en forme. Les thermoplastiques présentent une structure linéaire (ou légèrement ramifiée) enchevêtrée qui ne résulte pas d'une polymérisation. Lors de la mise en forme par chauffage, les macromolécules se replient puis se bloquent au cours d'un refroidissement. Pour le matériau obtenu après mise en œuvre, les liaisons entre macromolécules sont des liaisons physiques faibles de type Van Der Waals : cette transformation est réversible.

\subsection{Technologies de mise en forme des composites thermoplastiques}

Dans le marché automobile, on distingue entre deux types de procédés, la compression et l'injection :

\section{LFT : Long Fiber Technology}

LFT : Les granulés LFT sont réalisés par extrusion. Le polymère fondu et le renfort sous forme de fils coupés sont mélangés à des températures de l'ordre de $250{ }^{\circ} \mathrm{C}$. Le mélange est extrudé sous forme de joncs qui sont refroidis puis coupés. Les taux de verre peuvent aller de 10 à $60 \%$ en masse. Les granulés sont moulés sur des presses d'injection ou d'injection-compression où ils sont préchauffés à plus de $250{ }^{\circ} \mathrm{C}$. Ils sont injectés sous très forte pression (>200 bars) dans un moule fermé refroidi. Les temps de cycle varient suivant les pièces à réaliser de 30 secondes à 2 minutes 30. Les grands secteurs d'utilisation sont l'automobile, l'électrotechnique, l'électronique et l'électroménager, le matériel industriel est agricole.

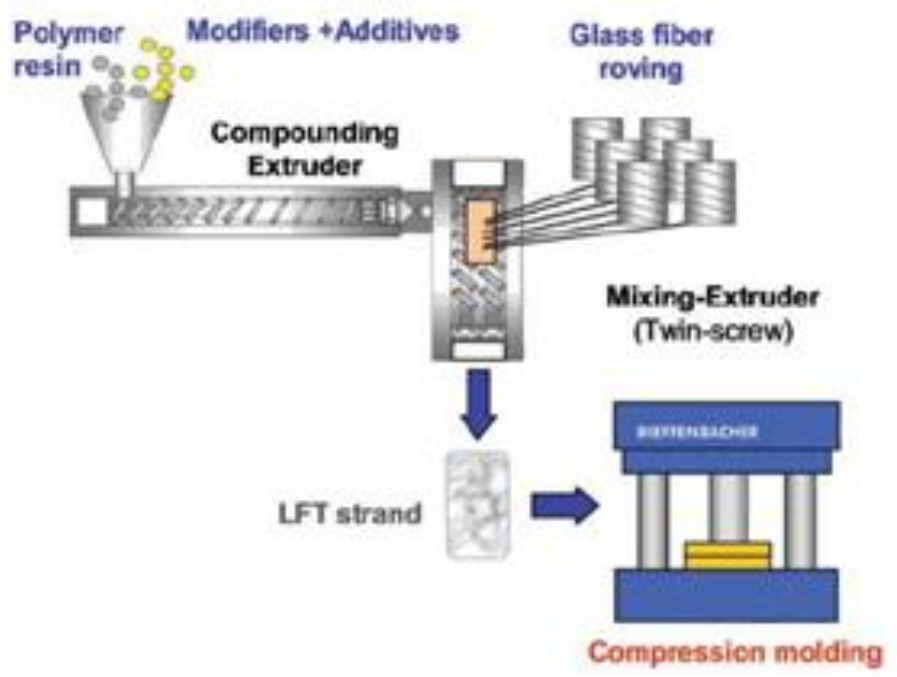

Figure 2. Schéma descriptif de la méthode LFT 
La compression de plaques GMT est essentiellement dédiée à l'automobile. Les matériaux en feuilles, réalisés généralement par extrusion/calendrage sont préchauffés sous forme de flans prédécoupés à plus de $250{ }^{\circ} \mathrm{C}$ (four Infra-Rouge) puis transférés dans un moule refroidi monté sur une presse verticale. Ils sont alors moulés par Compression / estampage. Les temps de cycle sont inférieurs à une minute mais le coût matière élevé les pénalise. Ils sont de plus en plus concurrencés par l'apparition de nouveaux couples matériaux / technologies du type LFT.

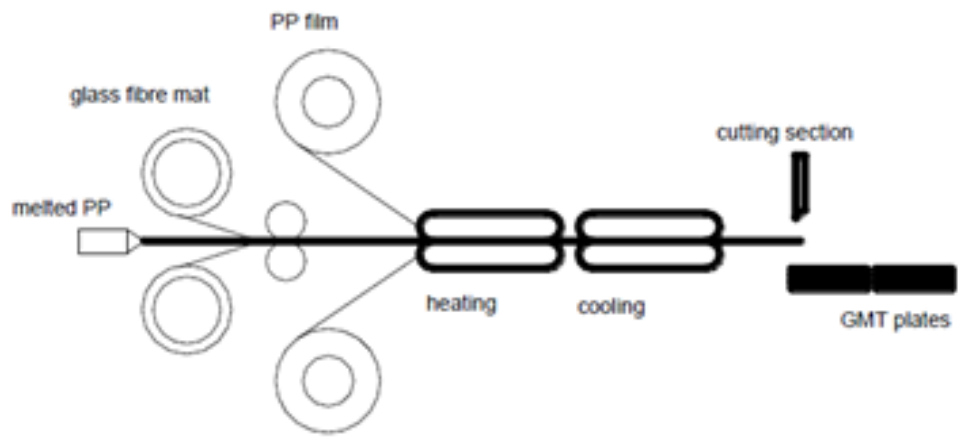

Figure 3. Schéma descriptif de la méthode GMT

\subsection{Caractéristiques mécaniques et thermiques des matériaux composites étudiés}

En générale les caractéristiques mécaniques ou statiques des matériaux composites utilisés dans l'automobile dépendent énormément sur le pourcentage et la nature des fibres utilisés dans le matériau.

Dans notre étude nous intéressons aux matériaux composites avec des fibres de verre continues et un pourcentage très élevé dans la matrice, ils sont réalisés à partir de la technologie LFT. Les caractéristiques mécaniques et thermiques du matériau étudié sont identifiés à partir des essaies physiques:

\begin{tabular}{|l|l|}
\hline critère & valeur \\
\hline Module de Young en traction & $30000 \mathrm{MPa}$ \\
\hline Coefficient de Poisson & $0,29 / 0,35$ \\
\hline Masse Volumique & $1,5 \mathrm{~g} / \mathrm{cm} 3$ \\
\hline Contrainte à la rupture (Rm) & $931 \mathrm{MPa}$ \\
\hline Allongement autorisé avant la rupture \% & $10 \%$ \\
\hline Module de Young en flexion & $31000 \mathrm{MPa}$ \\
\hline Contrainte à la rupture (Rm) en flexion & $606 \mathrm{MPa}$ \\
\hline $\begin{array}{l}\text { Allongement autorisé avant la rupture } \% \text { en } \\
\text { flexion }\end{array}$ & $\mathbf{8 \%}$ \\
\hline
\end{tabular}

Tableau 2. caractéristiques mécaniques du matériau étudié

Les courbes de contrainte en fonction de déformation ci-dessous ont été utilisés pour identifier les paramètres de la loi de JOHNSON-COOK, dans l'objectif est de modéliser numériquement le matériau composite avant de l'utiliser dans la simulation numérique.

La loi représente un modèle de comportement multiplicatif. Il prend en compte les effets de la vitesse de déformation, mais également ceux de la température. 


$$
\sigma(\varepsilon, \dot{\varepsilon})=\left(\sigma_{0}+K_{0} \varepsilon_{p}^{n}\right)\left(1+K_{1} \ln \dot{\varepsilon} / \dot{\varepsilon}_{0}\right)\left(1-T^{*_{m}}\right)
$$

Où $\mathrm{T}^{*}$ est une fonction de la température de fusion $\mathrm{Tm}$ et de la température de référence T0

$$
T^{*}=\frac{T-T_{0}}{T_{m}-T_{0}}
$$

K0 et $\mathrm{n}$ définissent l'écrouissage. K1 et $\dot{\varepsilon}_{0}$ définissent la sensibilité à la vitesse de déformation.

m coefficient de la sensibilité à la température.

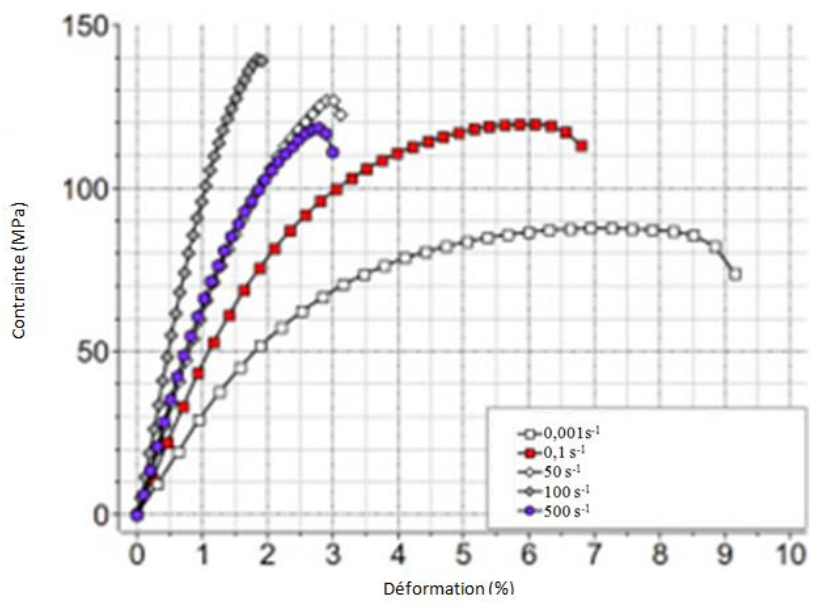

Figure 4. Courbes de contrainte en fonction de la déformation

\section{Application des composites dans l'automobile}

L'application des composites fibres longues concerne trois grands domaines dans les véhicules : bloc avant, bloc arrière, soubassement.

Bloc avant:

L'évolution des matériaux composites utilisés dans des pièces géantes montre les progrès effectués sur les matériaux et sur le savoir faire des constructeurs et des équipementiers.

\section{Car componentes made from LFT (1)}

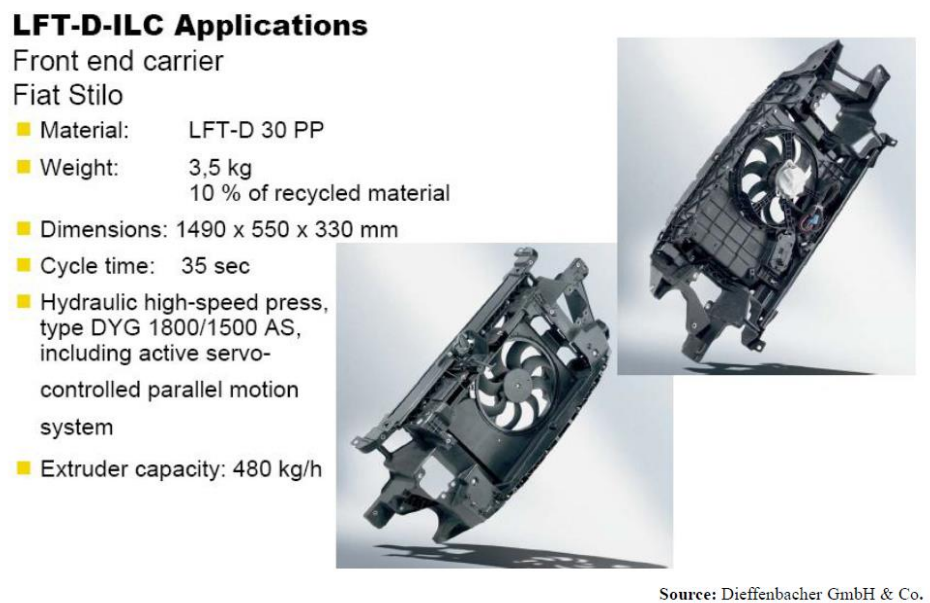

Figure 5. Image d'une façade bloc avant fabriquée en composite 

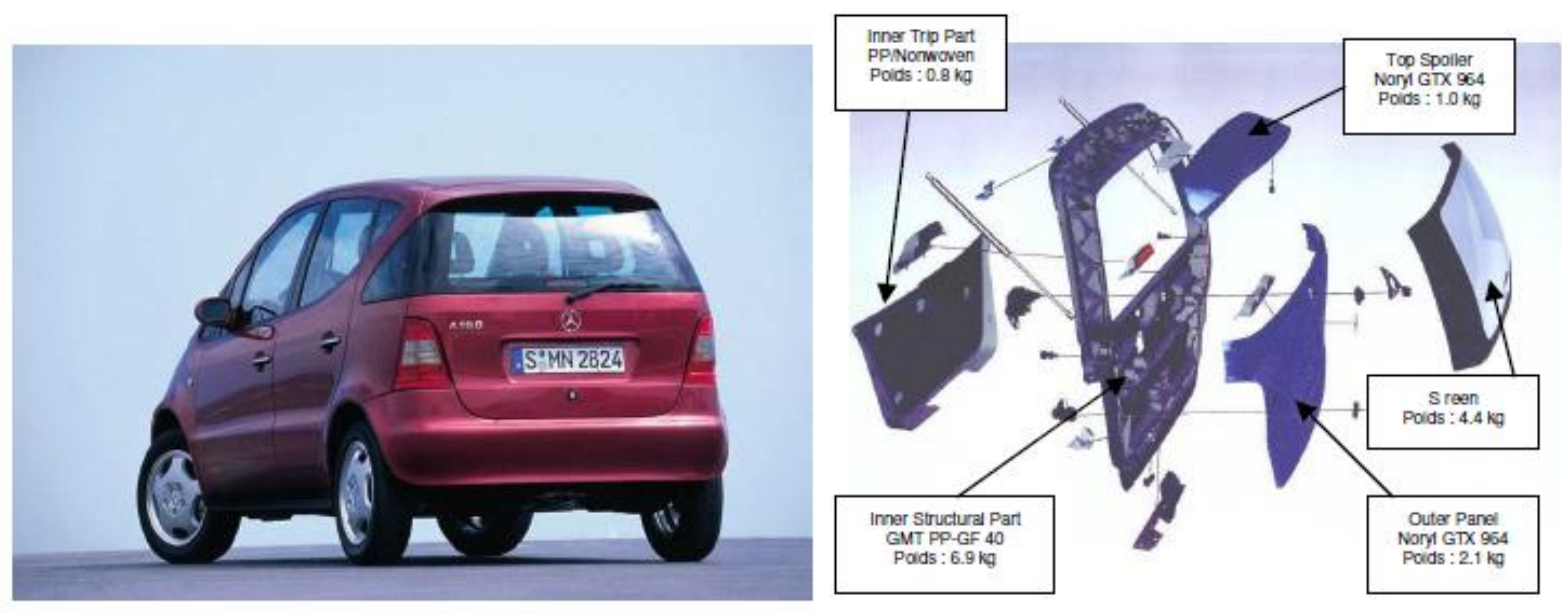

Figure 6. Image d'un hayon fabriqué en composite

\section{Soubassement :}

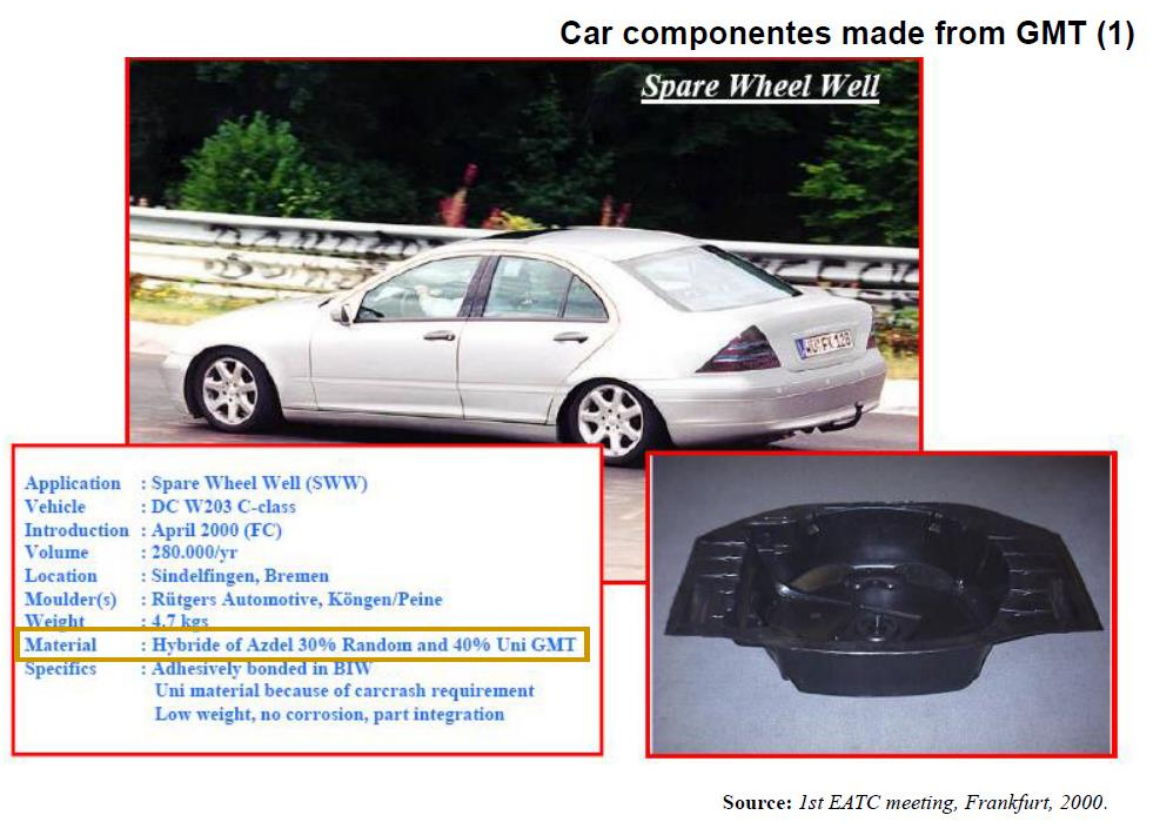

Figure 7. Image d'un plancher de charge fabriqué en composite

\section{Carrosserie :}

Le taux d'utilisation des matériaux composites dans les pièces de carrosserie automobile reste à ce jour encore assez marginal et on peut observer une forte disparité entre les constructeurs et selon les marchés.

\section{Evaluation des composites dans des pièces structurales}

Dans cette étude nous avons injecté ces matériaux dans des pièces structurales de l'automobile sollicités fortement dans les tests d'homologation, dans l'objectif est de tester numériquement ces matériaux en utilisant la simulation pour savoir le comportement et la fiabilité de ces matériaux. Le test que nous avons utilisé dans notre étude numérique est le ECE R14 ou FMVSS210 ; c'est un 
test utilisé pour vérifier les zones de fixation des ceintures de la sécurité au niveau de la carrosserie et les sièges.

La figure ci-dessous indique une illustration sur les moyens utilisés pour réaliser ce test :

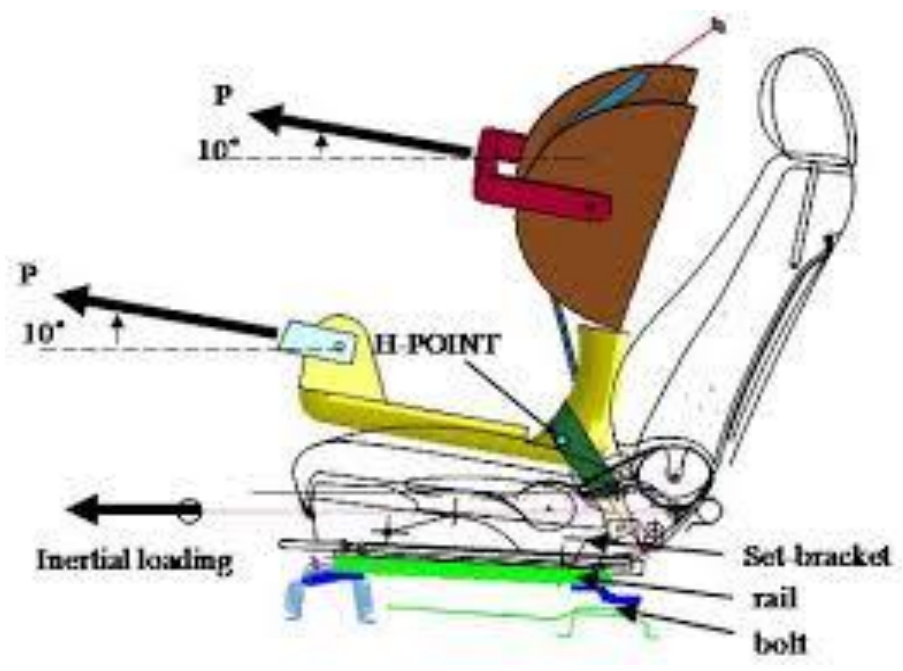

Figure 8. Test de résistance des ancrages de la ceinture

\subsection{Résultats de Comparaison avec l’acier}

L'étude numérique a été faite sur une pièce structurale de la carrosserie où on a fixé les sièges arrière via des liaisons boulonnées :

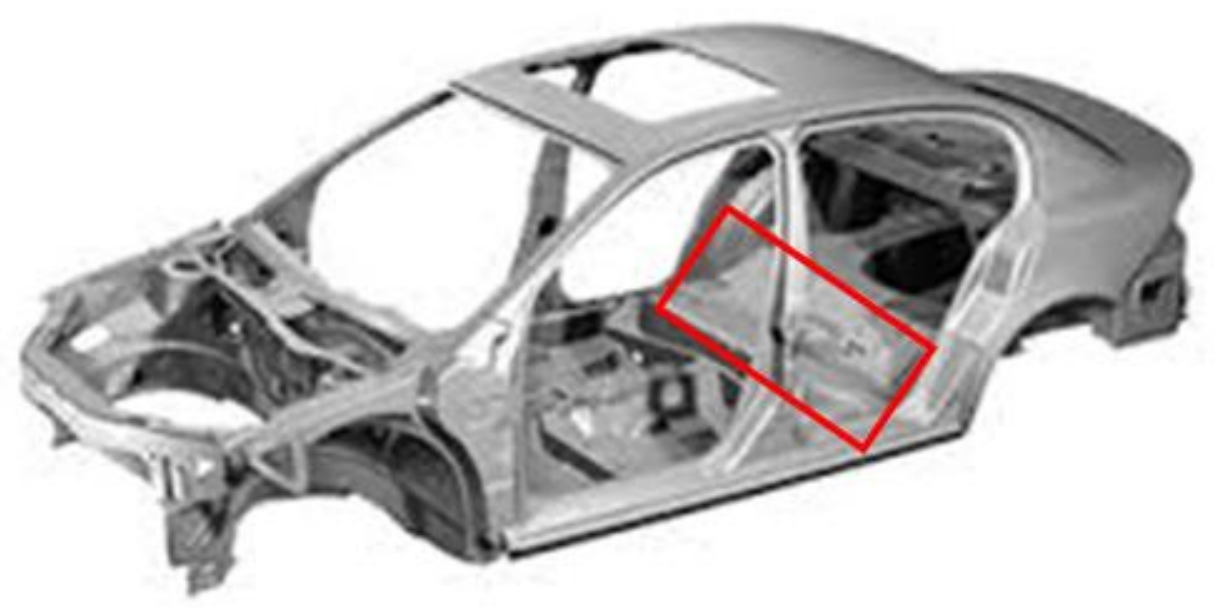

Figure 9. Zone de la pièce ciblée dans la carrosserie

Les résultats du calcul de la simulation numériques en utilisant le test de la résistance des ancrages de la ceinture montrent que les matériaux composites répondent favorablement aux sollicitations imposées lors de la simulation.

\subsubsection{Validation en déplacement}

Les résultats de déplacement en comparant entre l'acier et les composites montrent que ces derniers respectent suffisamment les valeurs critiques du test de l'homologation. 


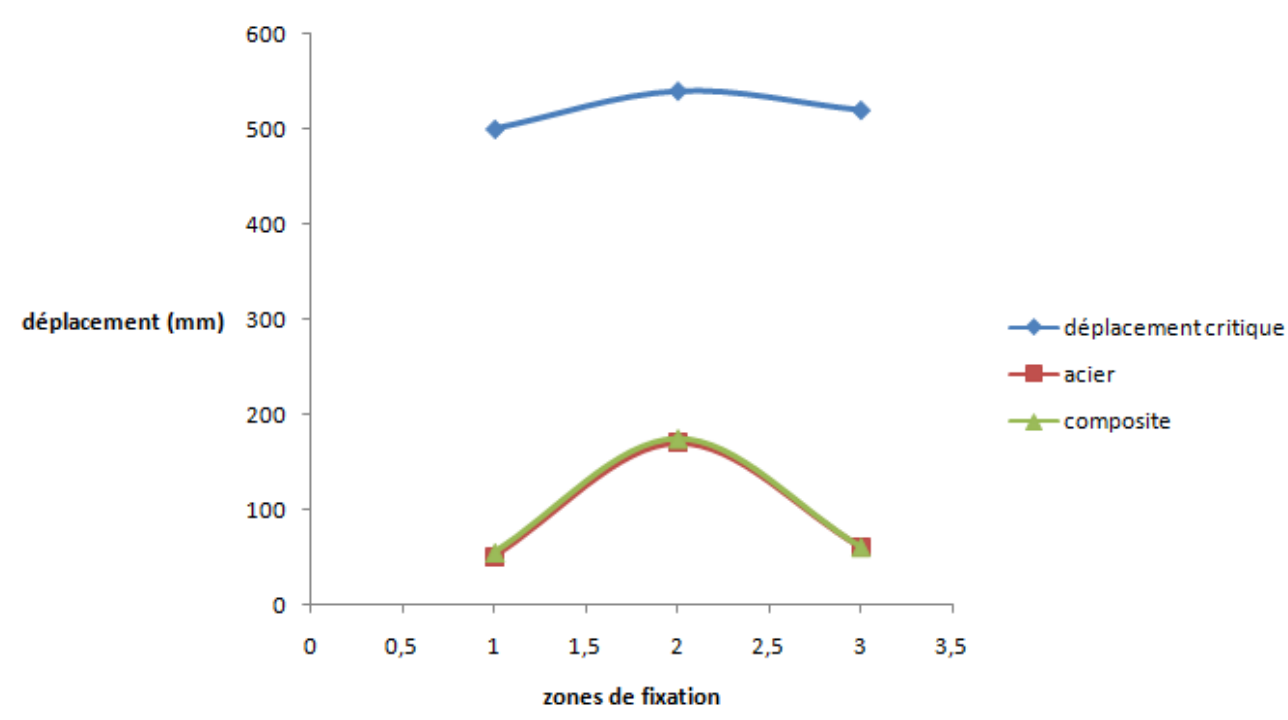

Figure 10. Courbes de déplacement

\subsubsection{Tenue mécaniques des composites}

En général dans un calcul quasi statique de l'automobile la validation de la tenue mécanique des pièces passent toujours par un calcul de plasticité en comparant avec les limites d'allongement des matériaux. Dans cette étude les résultats de la plasticité de notre matériau est favorable $(9,3 \%)$ par rapport à l'allongement autorisé de ce dernier (10\%), tout en gardant que la modification de la conception de la pièce ciblée par rapport à celle utilisée en acier, ainsi que les pièces qui l'entourent permettent d'améliorer énormément le comportement de la pièce dans la simulation.

\section{Conclusion}

Les résultats des travaux de la simulation numérique du test de l'homologation (résistance des ancrages de la ceinture) montrent que les matériaux composites thermoplastiques renforcés en fibres de verre continues sont capables de répondre aux critères de validation, pour des pièces sollicitées fortement au cours du test.

Aujourd'hui, ces matériaux représentent une solution innovante pour remplacer l'acier dans certaines pièces primordiales de la carrosserie, dans l'objectif final est de baisser le coût de fabrication et la consommation du gasoil.

\section{Bibliographie}

[1] J.Aucher, Etude comparative du comportement composite à matrice thermoplastique ou thermodurcissable. INSA de Rouen, 2009.

[2] Ecology and safety as a driving force in the development of vehicles. Radom, 2008.

[3] J.Carpenter \& Al, Research Needs in predictive engineering of advanced composite materials. 2004.

[4] M.Jeanne Dupont, A.Bentolila, B.Baroin, P.Bréard, The French industry of composite materials: priority challenges for sustainable development. DiGITIP Study. ISSN n 1241-1515.2001.

[5] Erica R.H , Strategic Material selection in the automobile body: Economic Opportunities for polymer composite design. Massachusetts Institute of Technology, 2006.

[6] Channel prime alliance. Celstran ${ }^{\circledR}$ CFR-TP PP GF70-13. Celanese Corporation- polypropylene. https://www.ulprospector.com , 2019 
[7] T.Renault, Les matériaux composites dans l'automobile. Faurecia Automotive Seating, 2001. 